\title{
Quantum-optical spectroscopy of a two- level system using an electrically driven micropillar laser as a resonant excitation source
}

Sören Kreinberg ${ }^{1}$, Tomislav Grbešić ${ }^{1}$, Max Strauß ${ }^{1}$, Alexander Carmele ${ }^{2}$, Monika Emmerling $^{3}$, Christian Schneider ${ }^{3}$, Sven Höfling ${ }^{3,4}$, Xavier Porte ${ }^{1}$ and Stephan Reitzenstein ${ }^{1}$

\begin{abstract}
Two-level emitters are the main building blocks of photonic quantum technologies and are model systems for the exploration of quantum optics in the solid state. Most interesting is the strict resonant excitation of such emitters to control their occupation coherently and to generate close to ideal quantum light, which is of utmost importance for applications in photonic quantum technology. To date, the approaches and experiments in this field have been performed exclusively using bulky lasers, which hinders the application of resonantly driven two-level emitters in compact photonic quantum systems. Here we address this issue and present a concept for a compact resonantly driven single-photon source by performing quantum-optical spectroscopy of a two-level system using a compact high- $\beta$ microlaser as the excitation source. The two-level system is based on a semiconductor quantum dot (QD), which is excited resonantly by a fiber-coupled electrically driven micropillar laser. We dress the excitonic state of the QD under continuous wave excitation, and trigger the emission of single photons with strong multi-photon suppression $\left(g^{(2)}(0)=0.02\right)$ and high photon indistinguishability $(V=57 \pm 9 \%)$ via pulsed resonant excitation at 156 $\mathrm{MHz}$. These results clearly demonstrate the high potential of our resonant excitation scheme, which can pave the way for compact electrically driven quantum light sources with excellent quantum properties to enable the implementation of advanced quantum communication protocols.
\end{abstract}

\section{Introduction}

The physics of two-level systems constitutes the basis for quantum optics and quantum cavity electrodynamics. It also has an important impact in the field of photonic quantum technologies, where it enables the secure exchange of information via single photons ${ }^{1-4}$ as well as efficient quantum computation with linear optics ${ }^{5}$. In particular, single photons are key resources for quantum

Correspondence: Stephan Reitzenstein (stephan.reitzenstein@physik.tu-berlin. de)

${ }^{1}$ Institut für Festkörperphysik, Technische Universität Berlin, 10623 Berlin, Germany

${ }^{2}$ Institut für Theoretische Physik, Technische Universität Berlin, 10623 Berlin, Germany

Full list of author information is available at the end of the article. key distribution using the BB84 protocol and for more advanced schemes, such as the quantum repeater concept for long-distance quantum communication. In such protocols and in quantum secure direct communication ${ }^{6}$, information is usually encoded in the polarization of the photon, and on-demand sources emitting single photons with high indistinguishability are of major importance for the implementation of these protocols. In this context, semiconductor quantum dots (QDs) are nearly ideal twolevel systems and can act as triggered sources of single photons ${ }^{7}$, where specific material properties can even be used for the direct generation of linearly polarized photons $^{8}$. To explore the physics of QDs and the quantum nature of emission, different excitation schemes have been

\section{(c) The Author(s) 2018}

(c) (i) Open Access This article is licensed under a Creative Commons Attribution 4.0 International License, which permits use, sharing, adaptation, distribution and reproduction cc. in any medium or format, as long as you give appropriate credit to the original author(s) and the source, provide a link to the Creative Commons license, and indicate if changes were made. The images or other third party material in this article are included in the article's Creative Commons license, unless indicated otherwise in a credit line to the material. If material is not included in the article's Creative Commons license and your intended use is not permitted by statutory regulation or exceeds the permitted use, you will need to obtain permission directly from the copyright holder. To view a copy of this license, visit http://creativecommons.org/licenses/by/4.0/. 
developed, which include simple non-resonant electrical and optical excitation as well as more advanced schemes, such as wetting-layer or $p$-shell resonant excitation ${ }^{9-14}$. Most interesting is the strict resonant excitation of the fundamental QD transition leading to resonance fluorescence $(\mathrm{RF})^{15-20}$. From an experimental point of view, strict resonant excitation is very demanding because it requires laser stray-light suppression by typically more than six orders of magnitude ${ }^{16,21,22}$. Nevertheless, the development of efficient suppression schemes and the availability of mode-hop-free tunable lasers have led to huge progress in this field, and RF has become an important experimental technique in quantum nanophotonics. For instance, strict resonant excitation has been used to study the subnatural linewidth from a single $\mathrm{QD}^{23}$ and to explore the non-resonant dot-cavity coupling in microcavity systems ${ }^{24}$. It is interesting to note that to date, the related experiments have only been performed using bulky and expensive laser systems.

In view of applications in quantum communications, strict resonant excitation of QDs is highly advantageous because it leads to the emission of single photons with excellent quantum properties in terms of multi-photon suppression and photon indistinguishability ${ }^{25}$. Both aspects are crucial for advanced quantum communication protocols based on entanglement distribution via Bellstate measurements ${ }^{26,27}$. In addition, to enable "realworld" applications, it is highly interesting to develop compact electrically driven quantum light sources. Unfortunately, standard excitation schemes based on carrier injection via a pin-diode design are intrinsically non-resonant, limiting the achievable degree of indistinguishability $^{28}$. To overcome this issue, an advanced excitation concept has been developed using an electrically driven microlaser to excite a single QD in a nearby microcavity system ${ }^{29}$. In this concept, quasi-resonant $p$ shell excitation was demonstrated ${ }^{30}$, but strict resonant excitation has not yet been achieved. In a similar scheme, a light-emitting diode was used for the on-chip excitation of a single $\mathrm{QD}^{31}$.

In this article, we demonstrate a fully nanophotonic approach to resonantly drive a QD acting as a two-level system and to generate single photons with excellent multi-photon suppression and a high degree of photon indistinguishability. Our concept is based on an electrically driven QD micropillar laser that resonantly drives a single QD located in a planar microcavity. To resonantly excite a two-level system, we use a microlaser spectrally matched to a QD, where the temperature of the microlaser is used as a fine-tuning knob in resonance scans. The experiments are performed under continuous wave $(\mathrm{CW})$ and pulsed excitation of the electrically driven microlaser to observe Mollow-triplet spectra and the triggered emission of single photons with a Hong-Ou-Mandel (HOM) visibility of $57 \%$, respectively. Our results show the potential of high- $\beta$ microlasers to act as excitation sources in quantum optics experiments and represent an important step toward the development of integrated quantum nanophotonic circuits relying on small-scale coherent light sources for resonant excitation of quantum emitters. This concept may lead to a significant cost reduction in quantum optics experiments when using microlasers instead of large laser systems as excitation sources. Even more interesting will be the application of low-threshold microlasers in integrated quantum circuits and compact quantum light sources, where they can

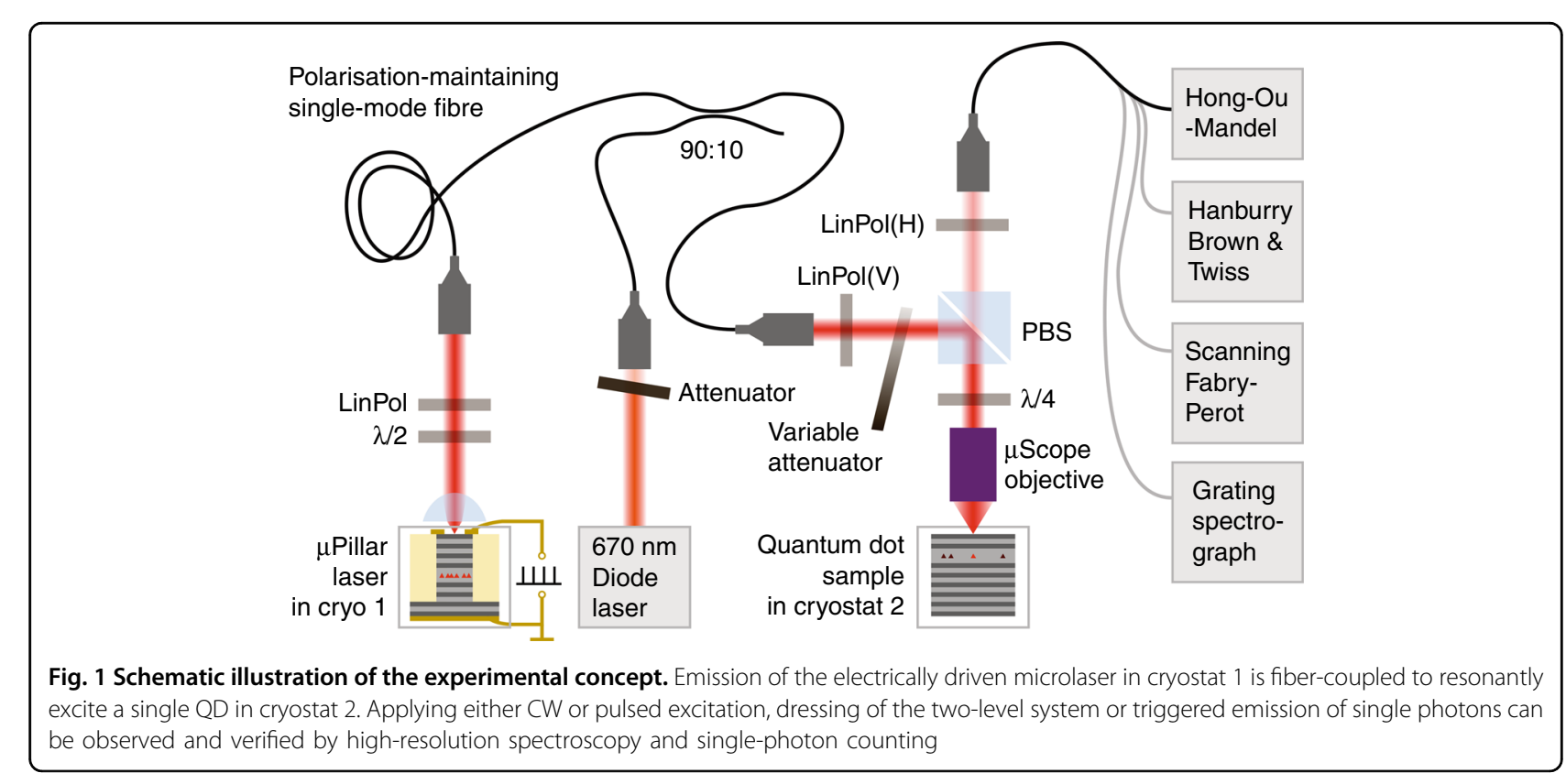


resonantly trigger the emission of single photons and photon pairs as key resources for photonic quantum technology.

Our experimental concept is illustrated in Fig. 1. It includes a QD micropillar laser located in cryostat 1 and a spectrally matched QD located in cryostat 2 . The light emitted by the microlaser is coupled into a $10 \mathrm{~m}$-long polarization-maintaining single-mode fiber, which is connected to the input port of the RF setup to excite the selected QD in cryostat 2. The microlaser is driven by an electrical voltage supply capable of delivering an adjustable DC bias and voltage pulses. The pulses have a width of $520 \mathrm{ps}$, an amplitude up to $8 \mathrm{~V}$, and a maximum repetition rate of $312.5 \mathrm{MHz}$. Resonance tuning with the tuning range of approximately $35 \mathrm{GHz}$ is enabled by changing the temperature of the microlaser in the 64-68 $\mathrm{K}$ range. The sample temperature of cryostat 2 is set to 7 $\mathrm{K}$ to minimize phonon-induced decoherence ${ }^{32}$ and carrier escape from the QDs in RF experiments. The need for cryogenic operation is also present for other relevant devices in quantum technology, such as superconducting nanowire single-photon detectors, and is in general required in advanced applications relying on quantum coherence, such as long-distance quantum communications networks. See the Materials and methods section for details on the sample technology and on the experimental setup.

For the planned quantum-optical studies, it is crucial to couple the emission of the high- $\beta$ microlaser with submicrowatt output power very efficiently into a singlemode fiber connecting the two cryostats. For this purpose, we collimate the microlaser emission via a single low-loss $f=20 \mathrm{~mm}$ aspheric lens in front of the optical window of cryostat 1 . In this context, we would like to note that a slight deviation of the circular cross section splits the fundamental transverse micropillar mode into two gaincoupled mode components with a spectral splitting of 11 $\mathrm{GHz}$. One of the two modes wins the gain competition and undergoes the lasing transition ${ }^{33,34}$. Emission of the lasing mode is selectively coupled into the polarizationmaintaining single-mode fiber via a combination of a halfwave plate plus linear polarizer and a collimating beam coupler.

\section{Materials and methods \\ Sample technology}

The QD microlaser and the resonantly excited QD are based on $\mathrm{AlGaAs}$ heterostructures grown by molecular beam epitaxy. Both structures consist of high-quality AlAs/GaAs-based distributed Bragg reflectors (DBRs) forming a planar microcavity with a central one- $\lambda$ GaAs cavity. A single layer of InGaAs QDs acts as the active medium. For the microlaser, the planar microcavity is composed of a rather high number (27 and 23) of mirror pairs in the $n$-doped (lower) and $p$-doped (upper) DBRs to ensure pronounced light-matter interaction and high- $\beta$ lasing. A dense array of micropillar lasers with a diameter of $3 \mu \mathrm{m}$ and a pitch of $60 \mu \mathrm{m}$ are realized by highresolution electron-beam lithography and subsequent reactive-ion etching. The sample is planarized with benzocyclobutene to mechanically support the ring-shaped upper $\mathrm{Au}$ contacts. This has the positive side effect of protecting the AlAs layers from oxidization. The realized array includes 62 electrically micropillar lasers emitting in the spectral range of $912-919 \mathrm{~nm}$. We refer to ref. ${ }^{35}$ for further details on the fabrication of electrically contacted micropillars. The QD sample used in the RF experiments has a more asymmetric microcavity design with 24 and 5 mirror pairs in the lower and upper DBRs, promoting directional outcoupling of light with an extraction efficiency of up to $42 \%^{36}$. Due to the low QD density of $2 \times$ $10^{9} \mathrm{~cm}^{-2}$ and the presence of random photonic defects, this planar microcavity sample is highly suitable for single QD experiments and does not require lateral device processing. To address only a single QD with our laser spot, we have selected a sample position with a very low local QD density.

\section{Experimental setup}

The experimental configuration consists of two independent helium-flow cryostats (cryostats 1 and 2, respectively), each placed on a different optical table. The electrically driven QD microlaser is installed in cryostat 1 , and a single-mode fiber guides the laser light to the RF configuration in a cross-polarization configuration ${ }^{18,19,21}$ at cryostat 2. The resonant laser light enters the RF setup via a fiber beamsplitter, where it is superimposed with light from a low-power non-resonant support laser. The latter is a red diode laser (emission wavelength: $670 \mathrm{~nm}$ ), the emission of which fills the charge traps adjacent to the QD to effectively gate the RF signal of the $\mathrm{QD}^{37}$. The combined lasers are collimated again to free space and are aligned with the RF detection beam path by a polarizing beamsplitter cube (PBS). Excitation of the QD and detection of RF is then performed confocally through a numerical aperture $=0.65, f=4 \mathrm{~mm}$ microscope objective. The main purpose of the PBS is to strongly suppress the laser light reflected from the sample. To compensate for possible polarization ellipticity and to maximize laser stray-light suppression, a quarter-wave plate is placed in the excitation/detection path between the PBS and the microscope objective ${ }^{19}$. The detected light is fed into a polarization-maintaining single-mode fiber, both for spatial filtering and to facilitate quantum optics experiments. For Hanbury Brown and Twiss and HOM-style singlephoton correlation experiments, superconducting singlephoton detectors with a time resolution full width at half maximum (FWHM) of $55 \mathrm{ps}$ are correlated. 
High-resolution RF spectra are recorded using a scanning Fabry-Perot interferometer with a spectral resolution of $100 \mathrm{MHz}$.

\section{Results and discussion}

In this work, we apply an electrically driven microlaser to demonstrate for the first time the high potential of micro- and nanolasers in quantum-optical spectroscopy. Indeed, while the research interest in miniaturized lasers has increased rapidly in recent years, their applicability as resonant excitation sources in quantum nanophotonics has been widely unexplored to date. To enable related studies under strict resonant excitation, it is crucial to obtain a microlaser that (a) can be operated electrically under CW and pulsed operation with an emission pulse width significantly shorter than the spontaneous emission lifetime (here $510 \mathrm{ps}$ ) of the QD, (b) shows single-mode emission with an emission linewidth significantly smaller than the homogeneous linewidth of approximately $1 \mathrm{GHz}$, (c) is spectrally matched with a target QD within the available temperaturetuning range on the order of $500 \mathrm{GHz}$, and (d) has sufficiently high optical output power of approximately $100-500 \mathrm{nW}$ at the single-mode fiber output to at least saturate the QD transition.

To meet these stringent requirements, we first performed reference measurements using a conventional tunable laser as the excitation source to select a QD showing pronounced and clean RF at $920 \mathrm{~nm}$ (see SI for more details on the reference measurement), where 920 $\mathrm{nm}$ corresponds to the central wavelength reachable by the micropillar lasers within the patterned array. All measurements shown in this paper are performed on this selected QD. In the second step, we chose a micropillar laser with a slightly shorter emission wavelength of 919 $\mathrm{nm}$ at $10 \mathrm{~K}$ so that it can be spectrally matched with the QD wavelength at $66 \mathrm{~K}$. Figure $2 \mathrm{a}$ shows the $32 \mathrm{~K}$ electroluminescence emission spectrum of the microlaser at the output of the single-mode fiber. Without any spectral filtering, we observe clear single-mode emission with a side-mode suppression ratio of $19 \mathrm{~dB}$ and no significant contribution from GaAs or wetting-layer emission (see $\mathrm{SI})$. Emission of the laser is coupled into a single-mode fiber, leading to the output power of $350 \mathrm{nW}$ (at $V_{\text {bias }}=$ $10.2 \mathrm{~V}$ ) at the fiber output.

Figure $2 \mathrm{~b}, \mathrm{c}$ presents the corresponding voltagedependent output power and spectral linewidth of the micropillar laser, respectively. The onset of laser action is indicated by the nonlinear increase of the output intensity between $V_{\text {bias }}=7$ and $8 \mathrm{~V}$ accompanied by a strong decrease of the emission linewidth to values well below $0.1 \mathrm{GHz}$. The associated transition from predominantly spontaneous emission to stimulated emission is confirmed by measurements of the bias voltage-dependent second-order photon autocorrelation function $g^{(2)}(\tau)$, which shows the typical bunching behavior in the threshold region with $g^{(2)}(0)>1$ and a transition toward coherent emission associated with $g^{(2)}(0)=1$ at high excitation $^{38,39}$. It is worth noting that the equal-time photon correlation only approaches $g^{(2)}(0)=1$ when the linewidth is already reduced by a factor of 100 , in accordance with ref. ${ }^{40}$. To avoid possible issues caused by the response time of the detectors, we attenuated the microlaser output in the $g^{(2)}(\tau)$ measurements above the threshold, keeping the count rate per detector always below $1 \mathrm{MHz}$.

Having fulfilled the requirements (a)-(d) discussed above, we are prepared for RF experiments using the selected QD micropillar laser as a coherent excitation source. For this purpose, the temperature of the fibercoupled microlaser in cryostat 1 is gradually varied between 64 and $68 \mathrm{~K}$, and emission of the QD in cryostat 2 is recorded via the attached RF setup. The corresponding emission spectra (under CW excitation) are presented in Fig. 3a as a color-scale intensity map. While only weak emission of the QD and strongly suppressed laser emission can be detected under resonant conditions for QD-laser detuning $>10 \mathrm{GHz}$, strong and very pronounced RF emission occurs at resonance. In fact, when scanning the microlaser emission over the QD $s$-shell resonance, a double-peak response with a splitting of $5 \mathrm{GHz}$ detuning can be resolved (cf. Fig. 3b). This splitting is attributed to the fine-structure splitting of the excitonic transition of the $\mathrm{QD}^{41}$. The measurements presented in Fig. 3a, b also indicate that the reflected laser light and the QD emission due to the above-band excitation by the red laser make only marginal contributions to the RF signal. In the present approach, both the microlasers and the QDs are fabricated to emit at similar wavelengths. Nevertheless, the fine tuning of the wavelengths required for $\mathrm{RF}$ is still a very demanding prerequisite that we overcome by selecting the appropriate microlaser and QD among many candidates. A more deterministic approach will require deterministic nanofabrication of suitable QDmicrocavity systems at the target wavelength ${ }^{42}$ and either electro-optical Stark tuning ${ }^{43}$ or more advanced straintuning $^{44}$ for spectral fine tuning of the QD.

Coherent excitation of the QD-based two-level system is demonstrated in Fig. 4. Through the attenuation of the micropillar laser emission, we cover a $\mathrm{CW}$ excitation power range of $35-400 \mathrm{nW}$. Figure 4a shows the corresponding emission spectra recorded with a highresolution Fabry-Perot scanning interferometer. With increasing excitation power, we observe the characteristic line broadening of the single emission line with a measured FWHM of $600 \mathrm{MHz}$ at low drive toward the evolution of the Mollow triplet at high excitation strengths ${ }^{45}$. The splitting of the outer lines of the Mollow triplet with 
respect to the center amounts to $640 \mathrm{MHz}$ at $350 \mathrm{nW}$. The occurrence of this important signature of coherent excitation is confirmed for the studied QD by reference measurements over a wider range of excitation powers using a standard tunable laser (see SI).

The quantum nature of RF emission is investigated by measuring the second-order photon autocorrelation function $g^{(2)}(\tau)$, again under CW excitation via the electrically driven microlaser. As seen in Fig. 4b, the excitation power-dependent photon correlation reveals pronounced antibunching statistics with strong suppression of multi-photon emission events associated with $g^{(2)}(0)<0.4$. Upon increasing the excitation power from 40 to $400 \mathrm{nW}$, the simple antibunching dip evolves into a periodically modulated autocorrelation function. The observed signatures are associated with Rabi oscillations in agreement with the Mollow triplet observed in the frequency domain (cf. Fig. 4a). Importantly, we observe $g^{(2)}(\tau)>1$ in the vicinity of zero time delay $\tau \approx 0$. This photon bunching increases slightly with increasing

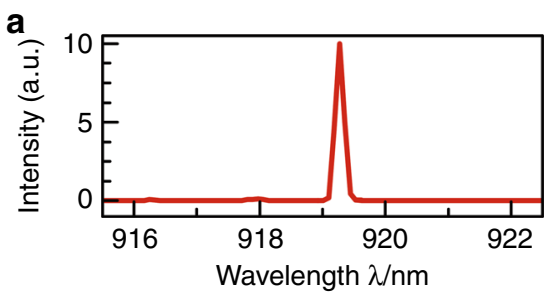

b

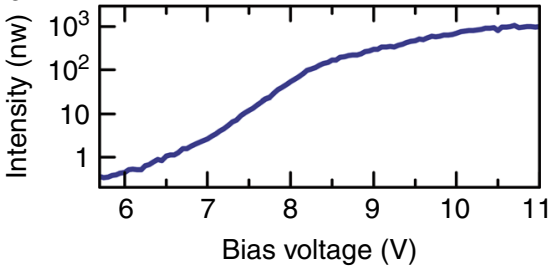

C

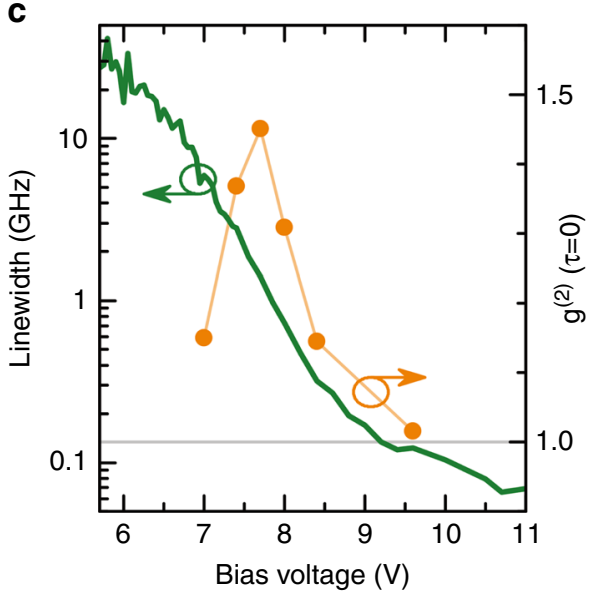

Fig. 2 Characterization of the electrically driven micropillar laser under CW excitation. a EL spectrum of the QD micropillar laser showing clean emission of the fundamental mode. Higher-order lateral modes of the micropillar are well suppressed (see SI for details). b Input-output dependence of the electrically driven QD micropillar laser with a threshold pump voltage of approximately 7-8V. c Equal-time second-order photon autocorrelation function (as measured) and spectral linewidth of the QD microlaser (deconvoluted taking the spectral resolution of the Fabry-Perot interferometer $(0.1 \mathrm{GHz})$ into account). The nonlinear input-output characteristics in conjunction with the narrowing of the emission linewidth by more than three orders of magnitude and the transition of $g^{(2)}(0)$ from values larger than one to unity are clear indications of predominantly stimulated emission of the QD microlaser above the threshold
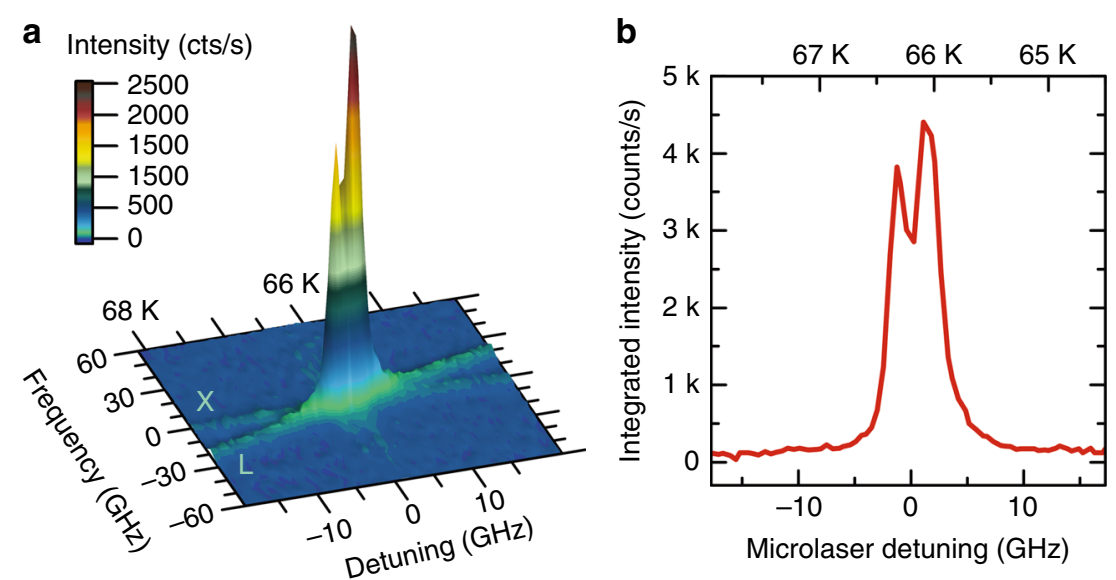

Fig. 3 Resonance fluorescence (RF) of a single QD under CW microlaser excitation $\left(\boldsymbol{V}_{\text {bias }}=\mathbf{1 0 . 2} \mathbf{V}\right)$. a 3D surface plot of the QD emission intensity as a function of the frequency $f$ and the microlaser detuning $\triangle$. Using temperature tuning in the range of $64-68 \mathrm{~K}$, the laser emission $(L)$ is tuned through the spectral resonance of the selected QD transition (X). A strong RF signal is observed in resonance at approximately $66 \mathrm{~K}$. $\mathbf{b}$ Emission intensity of the QD vs. laser detuning integrated over the spectral range of $-60 \mathrm{GHz} \leq f \leq 60 \mathrm{GHz}$ displayed in (a). The double-peak structure is attributed to the fine-structure splitting of the excitonic transition 

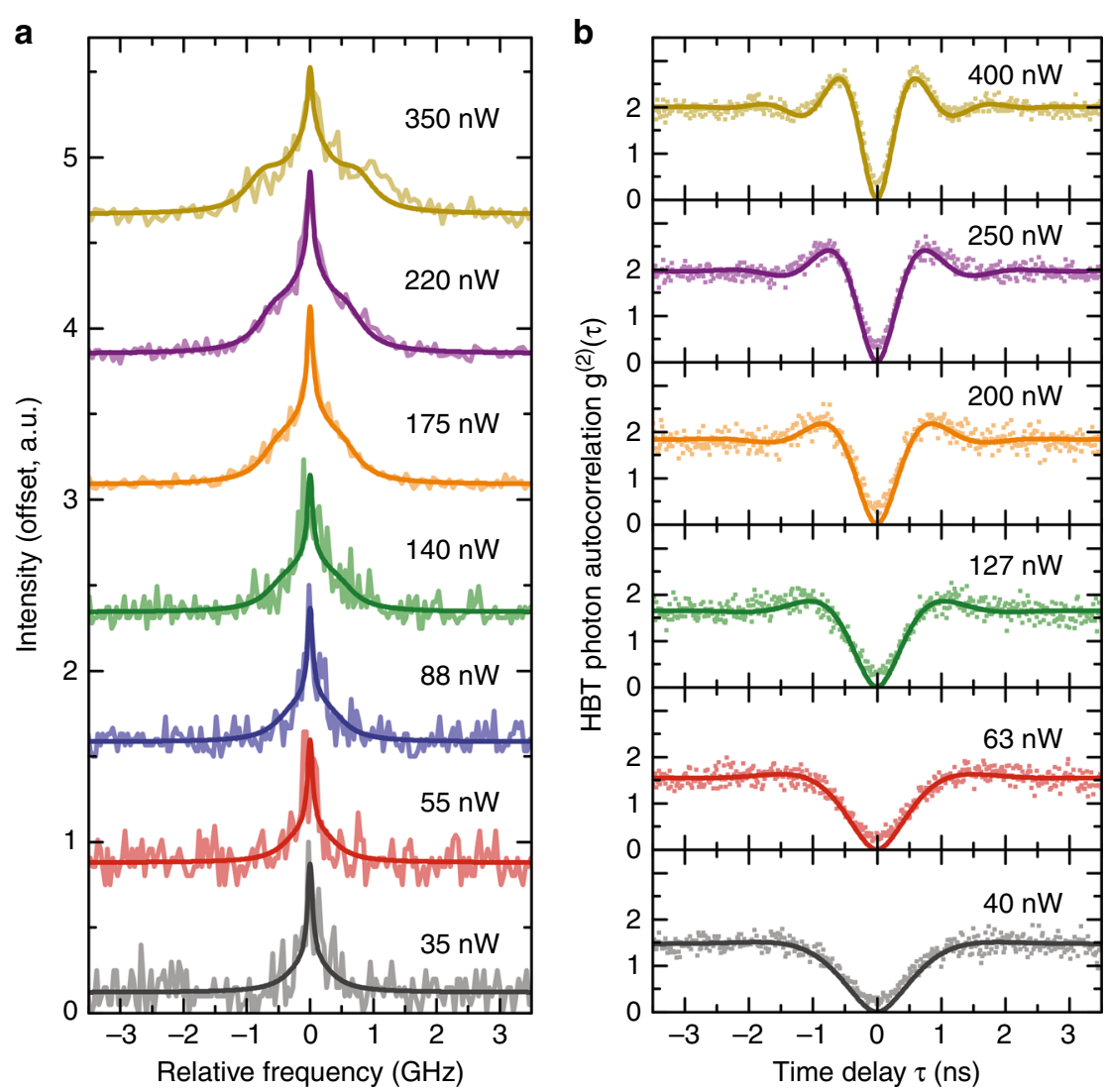

Fig. 4 Excitation-dependent resonance fluorescence (RF) emission spectra and photon autocorrelation function under CW microlaser excitation. a High-resolution RF emission spectra for different excitation powers. With increasing excitation power, we observe a transition of the single emission line toward a Mollow-triplet-like emission spectrum. b Second-order photon autocorrelation function $g^{(2)}(\tau)$ of the resonantly driven QD. The strong antibunching at zero time delay $\tau=0$ indicates single-photon emission. At higher laser powers, the narrowing of the antibunching peak together with the directly visible Rabi oscillations indicates coherent excitation of the two-level system

excitation power and indicates the blinking of the QD due to metastable processes ${ }^{46}$.

To obtain more detailed insight into the RF emission features and to theoretically describe the experimental data presented in Fig. 4 we consider the QD as a two-level system with a spontaneous emission lifetime $T_{1}$, a dephasing time $T_{2}$, and an excitation power-dependent Rabi frequency $\Omega . T_{1}=510 p s$ and $\Omega / \sqrt{P}=$ $2 \pi \times 1.33 \mathrm{THz} W^{-1 / 2}$ were determined via time-resolved experiments under pulsed micropillar laser excitation (cf. Fig. 5) and by investigating the excitation powerdependent autocorrelation using a standard tunable laser (see SI). Using the formulas introduced in the SI, we are able to model the experimental data under the variation of $T_{2}$. All optimal values of $T_{2}$ lie in the vicinity of $500 \mathrm{ps}$. Assuming $T_{2}=500 \mathrm{ps}$, we obtain excellent quantitative agreement between experiment and theory, as seen in Fig. 4a, b, where solid lines present the calculated data from formulas S3 and S4, respectively.

For applications in photonic quantum technology, it is crucial to demonstrate the triggered emission of single photons with excellent quantum properties. For this purpose, we biased the microlaser with $V_{\text {bias }}=4.71 \mathrm{~V}$ below the onset of lasing and superimposed voltage pulses with $V_{p p}=8 \mathrm{~V}$, a width of $520 \mathrm{ps}$, and a repetition period of $6.4 \mathrm{~ns}$. It is interesting to note that due to the nonlinear input-output dependence of the microlaser, the resulting optical emission pulses were shortened significantly to a width of 200 ps (FWHM). The ratio of the peak laser intensity to the strongest after-pulsing intensity was $>18$ $\mathrm{dB}$ (see SI). The pulsed emission was again coupled via the single-mode fiber in an RF configuration into cryostat 2 to resonantly excite the selected QD. The corresponding photon autocorrelation function was recorded at an excitation power of $22 \mathrm{nW}$ and is presented in Fig. $5 \mathrm{a}$. Pulsed emission of light is clearly identified by the train of correlation pulses separated by $6.4 \mathrm{~ns}$, and triggered single-photon emission is evidenced by the strongly reduced peak at zero delay with $g^{(2)}(0)=2 \%$. The zoomin presentation of $g^{(2)}(\tau)$ in the inset of Fig. 5a shows a characteristic substructure of the central $g^{(2)}(\tau)$ peak with a minimum at $\tau=0$ and side peaks at a finite delay. This 


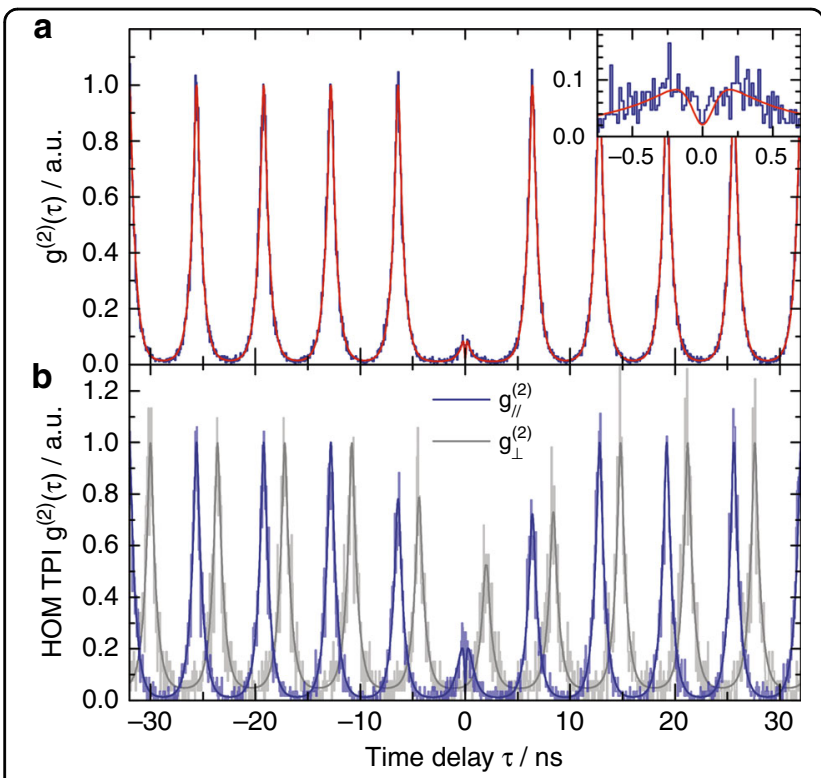

Fig. 5 Demonstration of triggered single-photon emission and photon indistinguishability under pulsed microlaser excitation. a Second-order photon autocorrelation under pulsed resonant excitation of the QD (pulse area: $0.9 \pi$ ). Triggered single-photon emission is clearly demonstrated by strong antibunching with $g^{(2)}(0) \ll 0.5$. The inset shows that the non-ideal $g^{(2)}(0)$ value can mainly be attributed to repeated QD excitation and decay within a single long-lasting laser pulse. This interpretation is confirmed by numeric modeling (red solid trace). b HOM histograms measured under co-polarized and crosspolarized (shifted by $\delta \tau=2 n s$ for the sake of clarity) configurations. Taking into account the non-ideal $g^{(2)}(0)$ value of the data presented in (a), we determine the HOM visibility to be $V_{\text {pure }}=0.57(9)$

correlation feature indicates that the non-ideal multiphoton suppression is mainly due to repeated QD excitation and decay within a single long-lasting laser pulse. Numerical modeling (red solid trace, cf. SI for details) was used to confirm the nature of the central correlation feature and to extract the lifetime of $T_{1}=510 \mathrm{ps}$ and the pulse area of $0.9 \pi$ by fitting the modeled curve to the experimental data. Indeed, it was predicted that both increased pulse length ${ }^{47}$ and increased pulse area ${ }^{48}$ increase the probability of multi-photon-photon events. Thus, in the future, even better multi-photon suppression may be achieved by applying shorter electrical pulses to the microlaser.

Finally, we study the photon indistinguishability of emission under pulsed microlaser excitation via a fibercoupled HOM two-photon interferometer with adjustable delay $^{32,49}$. Here the delay of the associated Mach-Zehnder interferometer was matched to the pulse repetition rate of $6.4 \mathrm{~ns}$ of the electrical voltage source used to drive the microlaser. The resulting photon correlation diagram of emission from the resonantly excited QD is displayed in Fig. 5b, both in the co-polarized and cross-polarized measurement configurations. The experimental data are displayed as light blue and light gray lines, and the numerically modeled fitting data (see SI) are displayed as dark blue and dark gray lines. The only fitting parameter (except for background counts and scaling) is the imbalance of the second beamsplitter, which is the splitter at which the HOM effect occurs, which is found to be 8:9 and results in the different heights of the peaks at $\pm 6.4 \mathrm{~ns}$. A significant degree of photon indistinguishability is evidenced by strongly reduced coincidences in the copolarized case, while for the cross-polarized case, we observe $g_{\perp}^{(2)}(0) \approx 0.5$, as expected for distinguishable photons. To determine the resulting two-photon interference visibility $V$, we first integrate the areas $A_{n}^{\|, \perp}$ of the peaks centered at time delays $\tau=n \times 6.4 n s, n \in$ $\{-7,-6,-5, \ldots, 6,7\}$ for each polarization configuration. Then, using

$$
\begin{gathered}
A_{r e f}^{\|, \perp}=\frac{1}{12} \sum_{n=2}^{7} A_{n}^{\|, \perp}+A_{-n}^{\|, \perp} \\
V=1-\frac{A_{0}^{\|} A_{r e f}^{\perp}}{A_{r e f}^{\|} f_{0}^{\perp}}
\end{gathered}
$$

we extract the raw two-photon interference visibility of $V=0.44(4)$. When compensating for the non-zero $g^{(2)}(0)$ and for the slight HOM beamsplitter imbalance of 8:9, we obtain a two-photon visibility as high as $V_{\text {pure }}=$ 0.57 (9) (see SI for details). This value is higher than the $41 \%$ reported in ref. ${ }^{28}$ for the direct non-resonant electrical excitation of a QD via carrier injection in a pindiode design. It is, however, significantly lower than the values exceeding $90 \%$ achieved by resonant excitation via standard mode-locked lasers with ps-pulse widths. Several possible effects can be considered in order to explain the non-ideal degree of photon indistinguishability, such as temperature-induced dephasing ${ }^{32,50}$ or spectral fluctuations $^{32}$. In the present case, i.e., under resonant excitation at low temperature, we can exclude these effects. Instead, we attribute the reduced HOM visibility mainly to the rather long optical pulse width of $200 \mathrm{ps}$ and to the nonFourier-limited dephasing time $T_{2}=0.5 n s \approx T_{1}<2 T_{1}$ (see Fig. S9). The increased laser pulse width in combination with strong pulse power $(0.9 \pi)$ leads to twophoton fluorescence pulses and in turn results in reduced HOM visibility ${ }^{47,48,51}$. On the other hand, the nonFourier-limited $T_{2}$ directly makes the photons more distinguishable either due to random phase changes or due to fine-structure splitting ${ }^{11,52}$ of the QD transition, thereby implying wavelength distinguishability. We therefore expect a strong improvement of the photon indistinguishability by carefully adjusting the detected polarization to a single QD transition only and by reducing the optical pulse length in future studies. 


\section{Conclusion}

In conclusion, we demonstrated a fully nanophotonic concept for the control of single-photon emission of a solid-state two-level system. The concept involves an electrically driven high- $\beta$ microlaser that resonantly drives a semiconductor QD acting as a two-level system. This work demonstrates for the first time the applicability of micro- and nanolasers in advanced quantum optics experiments under strict resonant excitation. Temperature-induced spectral fine tuning of a suitable QD microlaser allows us to observe the dressing of the fundamental QD transition and the occurrence of Rabi oscillations in photon correlation measurements. Pulsed electrical excitation of the microlaser leads to the emission of single photons with high multi-photon suppression $\left(g^{(2)}(0)=2 \%\right)$ and a HOM visibility as high as $57 \%$. As such, our results show the great potential of combining and coupling nanophotonic devices to systems with enhanced functionality. In the future, our concept could be further developed into a fully integrated on-chip resonantly pumped quantum light sources with many interesting applications in photonic quantum information technology. For instance, the implementation of quantum repeater networks will strongly benefit from sources of single and indistinguishable photons resonantly triggered by integrated microlasers instead of the use of standard large-scale laser systems as excitation sources.

\section{Acknowledgements}

The research leading to these results has received funding from the European Research Council (ERC) under the European Union's Seventh Framework ERC Grant Agreement No. 615613, the German Research Foundation (DFG) via CRC 787 and Projects No. RE2974/5-1, RE2974/9-1, and SCHN1376/2-1, the State of Bavaria, and the German Ministry of Education and Research (BMBF) within Q. com-H. A.C. gratefully acknowledges the support of the DFG through the project B1 of the SFB 910.

\section{Author details}

${ }^{1}$ Institut für Festkörperphysik, Technische Universität Berlin, 10623 Berlin, Germany. ${ }^{2}$ Institut für Theoretische Physik, Technische Universität Berlin, 10623 Berlin, Germany. ${ }^{3}$ Technische Physik, Julius-Maximilians-Universität Würzburg, 97074 Würzburg, Germany. ${ }^{4}$ SUPA, School of Physics and Astronomy, University of St Andrews, St Andrews KY16 9SS, UK

\section{Author contributions}

S.R. initiated the research and conceived the experiments. S.K. and T.G. performed the experiments. X.P. and S.R. supervised the experiments. A.C. did the continuous wave theoretical analysis. S.K. did the pulsed theoretical analysis. M.S. built the resonance fluorescence setup. M.E., C.S., and S.H. obtained the samples. S.R. and S.K. wrote the manuscript with contributions from all other authors.

\section{Conflict of interest}

The authors declare that they have no conflict of interest.

Supplementary information is available for this paper at https://doi.org/ 10.1038/s41377-018-0045-6.

Received: 15 May 2018 Revised: 6 June 2018 Accepted: 10 June 2018 Accepted article preview online: 27 June 2018

Published online: 25 July 2018

\section{References}

1. Gisin, N. \& Thew, R. Quantum communication. Nat. Photonics 1, 165-171 (2007).

2. Bouwmeester, D., Ekert, A. K. \& Zeilinger, A. The Physics of Quantum Information: Quantum Cryptography, Quantum Teleportation, Quantum Computation (Springer, Berlin, Heidelberg, 2000).

3. Nielsen, M. A. \& Chuang, I. L. Quantum Computation and Quantum Information (Cambridge Univ. Press, Cambridge, 2010).

4. Ladd, T. D. et al. Quantum computers. Nature 464, 45-53 (2010).

5. Knill, E., Laflamme, R. \& Milburn, G. J. A scheme for efficient quantum computation with linear optics. Nature 409, 46-52 (2001).

6. $\mathrm{Hu}, \mathrm{J}$. Y. et al. Experimental quantum secure direct communication with single photons. Light Sci. Appl. 5, e16144 (2016)

7. Aharonovich, I., Englund, D. \& Toth, M. Solid-state single-photon emitters. Nat. Photonics 10, 631-641 (2016).

8. Lundskog, A. et al. Direct generation of linearly polarized photon emission with designated orientations from site-controlled InGaN quantum dots. Light Sci. Appl. 3, e139 (2014).

9. Michler, P. et al. A quantum dot single-photon turnstile device. Science 290, 2282-2285 (2000)

10. Santori, C., Pelton, M., Solomon, G., Dale, Y. \& Yamamoto, Y. Triggered single photons from a quantum dot. Phys. Rev. Lett. 86, 1502-1505 (2001).

11. Santori, C., Fattal, D., Vučković, J., Solomon, G. S. \& Yamamoto, Y. Indistinguishable photons from a single-photon device. Nature 419, 594-597 (2002).

12. Ester, P. et al. p-Shell Rabi-flopping and single photon emission in an InGaAs/ GaAs quantum dot. Phys. E 40, 2004-2006 (2008).

13. Heindel, $T$. et al. Electrically driven quantum dot-micropillar single photon source with 34\% overall efficiency. Appl. Phys. Lett. 96, 011107 (2010).

14. Gschrey, M. et al. Highly indistinguishable photons from deterministic quantum-dot microlenses utilizing three-dimensional in situ electron-beam lithography. Nat. Commun. 6, 7662 (2015).

15. Muller, A. et al. Resonance fluorescence from a coherently driven semiconductor quantum dot in a cavity. Phys. Rev. Lett. 99, 187402 (2007).

16. Ates, S. et al. Post-selected indistinguishable photons from the resonance fluorescence of a single quantum dot in a microcavity. Phys. Rev. Lett. 103, 167402 (2009).

17. Vamivakas, A. N., Zhao, Y., Lu, C. -Y. \& Atatüre, M. Spin-resolved quantum-dot resonance fluorescence. Nat. Phys. 5, 925 (2009).

18. Unsleber, S. et al. Deterministic generation of bright single resonance fluorescence photons from a Purcell-enhanced quantum dot-micropillar system. Opt. Express 23, 32977-32985 (2015).

19. Strauß, M. et al. Photon-statistics excitation spectroscopy of a single two-level system. Phys. Rev. B 93, 241306(R) (2016).

20. Strauß, M. et al. Resonance fluorescence of a site-controlled quantum dot realized by the buried-stressor growth technique. Appl. Phys. Lett. 110, 111101 (2017).

21. Kuhlmann, A. V. et al. A dark-field microscope for background-free detection of resonance fluorescence from single semiconductor quantum dots operating in a set-and-forget mode. Rev. Sci. Instrum. 84, 073905 (2013).

22. Hopfmann, C. et al. Efficient stray-light suppression for resonance fluorescence in quantum dot micropillars using self-aligned metal apertures. Semicond. Sci. Technol. 31, 095007 (2016).

23. Matthiesen, C., Vamivakas, A. N. \& Atatüre, M. Subnatural linewidth single photons from a quantum dot. Phys. Rev. Lett. 108, 093602 (2012).

24. Ates, S. et al. Non-resonant dot-cavity coupling and its potential for resonant single-quantum-dot spectroscopy. Nat. Photonics 3, 724-728 (2009).

25. He, Y. M. et al. On-demand semiconductor single-photon source with nearunity indistinguishability. Nat. Nanotechnol. 8, 213-217 (2013).

26. Briegel, H. J., Dür, W., Cirac, J. I. \& Zoller, P. Quantum repeaters: the role of imperfect local operations in quantum communication. Phys. Rev. Lett. 81, 5932-5935 (1998)

27. Sangouard, N. et al. Long-distance entanglement distribution with singlephoton sources. Phys. Rev. A 76, 050301(R) (2007).

28. Schlehahn, A. et al. An electrically driven cavity-enhanced source of indistinguishable photons with 61\% overall efficiency. APL Photonics 1, 011301 (2016).

29. Munnelly, P. et al. Electrically tunable single-photon source triggered by a monolithically integrated quantum dot microlaser. ACS Photonics 4, 790-794 (2017). 
30. Stock, E. et al. On-chip quantum optics with quantum dot microcavities. Adv. Mater. 25, 707-710 (2013)

31. Lee, J. P. et al. Electrically driven and electrically tunable quantum light sources. Appl. Phys. Lett. 110, 071102 (2017).

32. Thoma, A. et al. Exploring dephasing of a solid-state quantum emitter via time- and temperature-dependent Hong-Ou-Mandel experiments. Phys. Rev. Lett. 116, 033601 (2016).

33. Leymann, H. A. M. et al. Intensity fluctuations in bimodal micropillar lasers enhanced by quantum-dot gain competition. Phys. Rev. A 87, 053819 (2013).

34. Redlich, C. et al. Mode-switching induced super-thermal bunching in quantum-dot microlasers. New J. Phys. 18, 063011 (2016)

35. Böckler, C. et al. Electrically driven high-Q quantum dot-micropillar cavities. Appl. Phys. Lett. 92, 091107 (2008).

36. Maier, S. et al. Bright single photon source based on self-aligned quantum dot-cavity systems. Opt. Express 22, 8136-8142 (2014).

37. Nguyen, H. S. et al. Optically gated resonant emission of single quantum dots. Phys. Rev. Lett. 108, 057401 (2012).

38. Ulrich, S. M. et al. Photon statistics of semiconductor microcavity lasers. Phys. Rev. Lett. 98, 043906 (2007).

39. Strauf, S. \& Jahnke, F. Single quantum dot nanolaser. Laser Photonics Rev. 5, 607-633 (2011).

40. Kreinberg, S. et al. Emission from quantum-dot high- $\beta$ microcavities: transition from spontaneous emission to lasing and the effects of superradiant emitter coupling. Light Sci. Appl. 6, e17030 (2017).

41. Bayer, M. et al. Fine structure of neutral and charged excitons in selfassembled In(Ga)As/(Al)GaAs quantum dots. Phys. Rev. B 65, 195315 (2002).
42. Dousse, A. et al. Controlled light-matter coupling for a single quantum dot embedded in a pillar microcavity using far-field optical lithography. Phys. Rev. Lett. 101, 267404 (2008).

43. Findeis, F., Baier, M., Beham, E., Zrenner, A. \& Abstreiter, G. Photocurrent and photoluminescence of a single self-assembled quantum dot in electric fields. Appl. Phys. Lett. 78, 2958-2960 (2001).

44. Trotta, R. et al. Nanomembrane quantum-light-emitting diodes integrated onto piezoelectric actuators. Adv. Mater. 24, 2668-2672 (2012).

45. Mollow, B. R. Power spectrum of light scattered by two-level systems. Phys. Rev. 188, 1969-1975 (1969).

46. Davanço, M., Hellberg, C. S., Ates, S., Badolato, A. \& Srinivasan, K. Multiple time scale blinking in InAs quantum dot single-photon sources. Phys. Rev. B 89, 161303(R) (2014).

47. Fischer, K. A., Müller, K., Lagoudakis, K. G. \& Vučković, J. Dynamical modeling of pulsed two-photon interference. New J. Phys. 18, 113053 (2016).

48. Fischer, K. A. et al. Signatures of two-photon pulses from a quantum two-level system. Nat. Phys. 13, 649-654 (2017).

49. Bennett, A. J. et al. Indistinguishable photons from a diode. Appl. Phys. Lett. 92 193503 (2008).

50. Varoutsis, S. et al. Restoration of photon indistinguishability in the emission of a semiconductor quantum dot. Phys. Rev. B 72, 041303(R) (2005).

51. Dada, A. C. et al. Indistinguishable single photons with flexible electronic triggering. Optica 3, 493-498 (2016).

52. Gazzano, O. et al. Bright solid-state sources of indistinguishable single photons Nat. Commun. 4, 1425 (2013). 